\title{
Les femmes dans les apparitions mariales de l'époque contemporaine
}

\author{
Marlène Albert-Llorca
}

\section{(2) OpenEdition}

1 Journals

Édition électronique

URL : https://journals.openedition.org/clio/64

DOI : $10.4000 /$ clio.64

ISSN : 1777-5299

Éditeur

Belin

Édition imprimée

Date de publication : 1 avril 2002

Pagination : 123-134

ISBN : 2-85816-620-X

ISSN : $1252-7017$

Référence électronique

Marlène Albert-Llorca, « Les femmes dans les apparitions mariales de l'époque contemporaine », Clio. Histoire, femmes et sociétés [En ligne], 15 | 2002, mis en ligne le 08 février 2015, consulté le 28 avril 2022. URL : http://journals.openedition.org/clio/64 ; DOI : https://doi.org/10.4000/clio.64 


\title{
Les femmes dans les apparitions mariales de l'époque contemporaine *
}

\author{
Marlène AlberT LLORCA
}

Dans son article sur la "féminisation du catholicisme» au XIX" siècle, Claude Langlois rappelle que ce siècle est «celui des apparitions mariales». Et il ajoute : «Mais c'est également celui des voyantes» ${ }^{1}$. Cette affirmation peut s'entendre de deux manières qui n'ont, du reste, rien de contradictoire. On peut lui donner un sens statistique : au XIX'siècle, les bénéficiaires des apparitions mariales auraient été plus souvent des femmes que des hommes. On peut aussi la référer aux attitudes de l'Église à l'égard des apparitions où les visionnaires furent des femmes'. On songe en particulier à celles de Lourdes, que l'évêque de Tarbes déclara authentiques dès le 18 janvier $1862^{3}$. Cette approbation contribua sans doute à suggérer que

* Je remercie W. A. Christian d'avoir relu cet article et de m’avoir donné son uimprimatur".

1 Langlois 1991 : 295. La remarque vaut surtout pour la seconde moitié du XIX siècle, les apparitions restant rares, comme le soulignent Bouflet et Boutry (1997 : 104), durant les premières décennies du siècle.

2 J'utiliserai indifféremment les termes voyant(e) et visionnaire pour désigner les bénéficiaires des apparitions.

3 Deux autres apparitions du XIXe siècle ont été déclarées authentiques dans les mois ou les années qui les suivirent : La Salette (1846, reconnue en 1851); Pontmain (1871,

CLIO, Histoire, Femmes et Societés, 2002, 15, pp. 123-134. 
les femmes étaient aussi qualifiées que les hommes, voire plus qu'eux, pour voir la Vierge. En s'appuyant sur les données fournies par J. Bouflet et $P$. Boutry $y^{4}$ sur vingt-huit cas d'apparitions survenues entre $1846^{5}$ et la fin du siècle, on constate en effet que les voyantes sont deux fois plus nombreuses que les voyants ${ }^{6}$.

Pour pouvoir conclure, de ces chiffres, à une "féminisation de la voyance" - à mettre en relation, j'y reviendrai, avec la "féminisation du catholicisme» - il faut s'assurer cependant que les femmes n'ont pas tenu une place aussi importante dans les apparitions des siècles précédents. Les données dont nous disposons sur les apparitions du XIX siècle invitent, par ailleurs, à préciser les modalités de cette féminisation. Elles montrent en effet que les «femmes» impliquées dans les apparitions de ce siècle sont presque toujours des jeunes filles ou des fillettes. Ce constat, au demeurant, ne vaut pas que pour le XIXe siècle mais aussi pour les premières décennies du suivant, ce qui conduit à s'interroger sur les aspects de la féminité valorisés dans le catholicisme.

\section{Voyantes et mystiques}

Avant de donner les résultats des enquêtes effectuées sur l'identité sexuée des voyants, pour les apparitions de la Vierge antérieures au XIX ${ }^{c}$ siècle, il faut préciser qu'il ne sera ici question que des bénéficiaires d'ap-

reconnue en 1872). D'autres apparitions de ce siècle furent reconnues seulement au $\mathrm{xx}$.

4 Bouflet et Boutry 1997 : 127-202. J'ai utilisé le livre de Chiron (1995) lorsque celuici donnait des informations plus précises sur l'identité des voyants. Ces auteurs traitent surtout des apparitions qui se sont produites en France.

51846 est la date des apparitions de La Salette. Sur leur importance dans l'histoire des apparitions, cf. Bouflet et Boutry $1997: 127$.

6 Ce résultat ne tient pas compte des cas où l'on mentionne plusieurs adultes ou enfants, sans autre précision, ni des cas où la Vierge apparut à un nombre indéterminé de fillettes : à Sassay (1873), une classe de caréchisme ; à Tilly sur Seulles (1896), les filles d'une école religieuse et plusieurs de leurs enseignantes. Je n'ai, enfin, comptabilisé que les quatre premières fillettes à qui la Vierge apparut à Neubois (1872). Or, selon G. Lameire (1978), il y eut au total 121 voyantes sur les 147 voyants qu'il a identifiés. 
paritions et non de visions. J. Bouflet et $P$. Boutry définissent ainsi l'une et l'autre notion. Une apparition est «une révélation particulière, mais publique dans sa manifestation et ses effets" ${ }^{7}$. La Vierge et les saints - le Christ est beaucoup plus rarement concerné - sont généralement apparus, pour délivrer un ou des messages. Ceux-ci sont destinés à la communauté locale à laquelle appartenait le visionnaire : construire une chapelle, organiser des processions destinées à appeler la grâce de Dieu. Ils peuvent aussi être destinés à la nation où se produit l'apparition. À Ezkioga (Pays basque espagnol) en 1931, la Vierge aurait appelé à "sauver l'Espagne» qui venait de se doter d'un régime républicain. Le message peut enfin s'adresser à la catholicité ou à l'humanité tout entière; c'est le cas dans les "secrets" de La Salette ou de Fatima et, plus généralement, dans la majorité des messages délivrés au cours des apparitions mariales du XXe siècle. Une vision, en revanche, est «une révélation particulière privée, qui ne concerne que le récipiendaire» ${ }^{8}$. Le fait d'avoir des visions est une des caractéristiques de la sainteté mystique (ce qui ne signifie pas qu'il constitue, aux yeux de l'Église, un critère de sainteté), les visions y étant "considérées comme étapes dans l'ascension mystique» du sujet qui les reçoit?

Les visions, certes, ne demeurent pas toujours "privées". La majorité des mystiques ont mis par écrit leurs expériences, ce qui a sans doute eu pour effet d'éveiller ou de renforcer des vocations religieuses ${ }^{10}$. On sait aussi que certaines de leurs révélations avaient une dimension prophétique et, enfin, que leurs visions ont parfois donné naissance à de nouvelles dévotions, par exemple le culte du Sacré Cour ${ }^{11}$. Les visions peuvent donc avoir des effets publics. Mais, même dans ce cas, on ne saurait les identifier aux apparitions qui se traduisent toujours par l'émergence d'un nouveau lieu de culte - durable ou non - ou, parfois, la réactivation d'un ancien site cultuel. L'identité sociale des bénéficiaires des visions et des apparitions differe également. La majorité des mystiques (qui sont

\footnotetext{
7 Bouflet et Boutry 1995: 10

8 Bouflet et Bourry 1995: 10.

9 Maître 1997 : 88.

10 Albert 1997 : ch. 9.

11 Cf. Vauchez 1999 et Maître 1997.
} 
presque toujours des femmes) étaient des religieuses ou, du moins, appartenaient à un Tiers-ordre ; à de rares exceptions près, les voyants des apparitions mariales sont des laïcs.

\section{Voyantes et voyants}

Que savons-nous, donc, de l'identité sexuée de ces personnages pour les siècles antérieurs au XIX ${ }^{\complement}$ ? Pour répondre à cette question, je m'appuierai essentiellement sur les travaux de l'historien et anthropologue W. A. Christian. Ils sont centrés sur l'Espagne mais couvrent toute l'histoire des apparitions dans ce pays ${ }^{12}$ ou, plus précisément, des apparitions attestées par des documents contemporains de l'événement ${ }^{13}$ : pour le Moyen Âge et le début de la période moderne, les enquêtes effectuées par les autorités civiles et religieuses locales, puis les procès d'Inquisition; pour l'époque contemporaine, les archives civiles et religieuses auxquelles s'ajoutent les articles de presse, les écrits des témoins, etc. La première apparition attestée par un document de ce type date de 1399 ; plusieurs autres se produisent au XV' siècle. Ce premier cycle s'arrête en Castille au

12 Ces travaux ont donné lieu à trois livres $(1981,1992,1996)$ consacrés à une étape de cette histoire. Christian a également écrit un article sur les apparitions de la Guerre Froide (1984) et il donne quelques indications dans un autre article (1998) sur les apparitions postérieures au Concile Vatican II. Les références aux livres initialement publiés en 1981 et 1996 renvoient à leur traduction en espagnol (1990 et 1997).

13 Les légendes médiévales de fondation des sanctuaires attribuent parfois leur origine à une apparition mais on ne saurait, comme le dit Christian (1990:18), mettre sur le même plan ces légendes, écrites à des dates bien postérieures, et les textes contemporains de l'apparition. Car ceux-ci, à la différence des premiers, nous permettent de savoir qui étaient les voyants, quel était le contenu de leurs visions, comment la population et les clercs reçurent leurs déclarations, etc. Christian souligne néanmoins que les légendes de fondation ont certainement influencé la construction des récits d'apparition et la créance qu'on leur a accordée. Le stéréotype du berger voyant en fournit un exemple. Pratiquement toutes les légendes médiévales rattachent la fondation des sanctuaires mariaux (presque toujours situés à l'écart des agglomérations) à la découverte miraculeuse, par un berger, de l'image sainte qui y est vénérée (et non à une apparition). Aussi a-t-on volontiers prêté foi aux bergers qui disaient avoir vu la Vierge et fait de Bernadette une bergère. 
début du XVI ciècle, du fait de la répression exercée par l'Inquisition ${ }^{14}$. Du $\mathrm{XVII}^{\mathrm{c}}$ au début du XXc siècle, les apparitions vont laisser place à un autre type de manifestations du surnaturel : des images saintes (des Christ notamment) qui pleurent, saignent, etc. ${ }^{15}$. Dans les semaines qui suivent la proclamation de la Seconde République espagnole, le 14 avril 1931, plusieurs apparitions se produisent dans le nord du pays : celles d'Ezkioga, dans le Pays basque, vont attirer des foules considérables ${ }^{16}$. Un nouveau cycle s'ouvre alors, qui va durer tout au long du siècle.

Dans un de ses derniers articles, Christian a rassemblé les données qu'il a recueillies sur l'identité sexuée et l'âge des visionnaires espagnols de la fin du Moyen Âge au début du XX siècle. Dans les apparitions survenues entre 1399 et 1592 , on trouve trois fois plus de voyants que de voyantes, tous âges confondus ${ }^{17}$. Au XIX ${ }^{e}$ siècle, comme le montrent les chiffres cités précédemment, la proportion des hommes et des femmes s'inverse. Précisons cependant que, sur les trente-deux voyantes identifiées dans la documentation fournie par Bouflet et Boutry, il n'y a que six femmes de plus de vingt ans, toutes les autres étant des fillettes ou des adolescentes $^{18}$. Et, parmi les six femmes, on trouve quatre célibataires, une veuve d'une trentaine d'années et une jeune femme de vingt-six ans dont j'ignore si elle était mariée ou non ${ }^{19}$. Sur ce point, les choses n'ont guère évolué depuis le Moyen Âge où l'on compte sept femmes mariées sur les vingt-sept voyantes mentionnées dans les documents.

Aucune évolution, non plus, entre le XIX siècle et l'Espagne des années 1930. Précisons, avant de livrer les données recueillies par Christian sur

14 Christian 1990 : ch. 3. Quelques cas sont attestés ensuite en Catalogne. En Italie, on en trouve jusqu'à la fin du XVI' siècle (Christian 1990 : 199). Ensuite, les apparitions s'arrêtent presque complètement jusqu'à la fin du XIX' siècle.

15 Christian 1992.

16 Christian 1997.

17 Christian $1998: 114$. Les chiffres donnés sont les suivants : 40 hommes, 33 garçons, 20 filles et 7 femmes.

18 J'ai classé dans la première catégorie les filles âgées de 12 ans ou moins (on en trouve 18).

19 Mary Mc Loughlin, une des deux premières voyantes de Knock (1879, Irlande). Cf. Chiron $1995: 260$. 
les visionnaires d'Ezkioga, que certains d'entre eux eurent des visions pendant plusieurs années : ils continuèrent à "voir" en dehors d'Ezkioga après que les pouvoirs civils puis religieux eurent interdit l'accès au lieu des apparitions ${ }^{20}$. La nouvelle des apparitions suscita en outre une sorte d'épidémie visionnaire : entre 1931 et 1936 , Christian a identifié 250 personnes qui eurent des visions, soit à Ezkioga, soit dans d'autres localités du Pays basque ou de Navarre - et il pense qu'il y en eut beaucoup plus. Le cas n'a rien d'exceptionnel. À Lourdes, cinq femmes virent la Vierge à Massabielle à partir du 7 avril et, entre mai et juillet, de nombreux enfants eurent aussi des visions, à Massabielle ou ailleurs ${ }^{21}$. Des "épidémies» analogues, mais de plus grande ampleur, eurent lieu, en Alsace, dans les mois qui suivirent les apparitions de Neubois (1872) et, en Belgique, celles de Beauraing (1932) 22. Ce qui est plus rare, en revanche, c'est qu'un chercheur se soit attaché à préciser l'identité des dizaines de voyants impliqués dans ces vagues d'apparitions. Christian l'a fait en s'appuyant à la fois sur les archives de la commission d'enquête chargée d'examiner les voyants, la presse, les photographies prises par les journalistes et les pèlerins, les témoignages oraux des habitants d'Ezkioga et des villages environnants. La confrontation de ces différentes sources montre que les membres de la commission d'enquête et les journalistes ne prêtèrent pas une égale attention à tous les visionnaires. Voyons qui sont ceux que l'on crut et ceux que l'on ignora.

La commission, qui comprenait les deux prêtres d'Ezkioga, son maire et le secrétaire de mairie, un médecin et un pharmacien, accueillit avec faveur les déclarations des hommes : "En général, on accorde peu de crédit aux visions des femmes (pardon, du beau sexe). Les déclarations d'hommes forts et robustes, tout comme celles des enfants, font réfléchir profondément ${ }^{23}$. Les clercs médiévaux et ceux qui leur succédèrent

20 Les autorités civiles intervinrent à la fin de l'année 1932 ; l'évêché, qui avait manifesté des réticences à l'égard des apparitions dès la fin de l'année 1931, leur dénia tout caractère surnaturel le 15 septembre 1934 (Christian $1997: 8$ et 9).

21 Harris 2001 : 134-154.

22 Bouflet et Boutry 1995 : 173-178 ; 238-239.

23 Cité par Christian $1997: 251$. 
avaient une opinion très semblable sur les femmes. Dans le traité De probatione spirituum (Du discernement des esprits) écrit en 1415, Jean Gerson appelait à se méfier des femmes visionnaires : "pleines de curiosité, elles sont comme celles que décrit l'Apôtre [saint Paul] : des petites bonnes femmes (mulierculae) chargées de péchés et agitées de toutes sortes de passions ${ }^{24}$. Les enfants, au contraire, sont présumés innocents et c'est sans doute pour cette raison qu'on les croyait aptes à entrer en contact avec le monde surnaturel : dans les apparitions médiévales documentées, ils sont (tous sexes confondus) légèrement plus nombreux que les adultes $^{25}$. Ils furent également écoutés en 1931 et les journaux leur ouvrirent largement leurs colonnes : "la presse rendit compte de quatre-vingt douze visions d'enfants (neuf filles et huit garçons) âgés de trois à quatorze ans $»^{26}$.

Le filtrage opéré par la commission se répercuta en effet sur la presse, les journalistes interrogeant de préférence les voyants qu'elle avait jugés crédibles. Pendant le premier mois, presque aucun homme n'eut de visions répétées. Pourtant, "la presse publia des photographies de sept des vingt-trois hommes adultes [qui furent interrogés] ${ }^{27}$. En revanche, aucune voyante ne fut photographiée et il en alla de même par la suite : au total, les journaux publièrent vingt-huit photographies d'hommes mais aucune de femme. Les autres sources étudiées montrent pourtant que le groupe des "voyants réguliers» (habitual seers) compte trois fois plus de femmes adultes que d'hommes ${ }^{28}$. Le cas de Maria, une voyante de Vizcaya, est très significatif de la censure exercée par la presse. Lorsqu'elle arriva à Ezkioga, elle avait 37 ans, était mariée et avait six enfants, dont un était né avant son mariage :

24 Je cite d'après Christian $1990: 245$.

25 Cf. les chiffres cités dans la note 16.

26 Christian $1997: 253$.

27 Christian $1997: 251$. L'auteur précise (1997:259) qu'il classe parmi les adultes les hommes de plus de 25 ans et parmi les jeunes filles, les filles de 15 ans ou plus.

28 Christian $1998: 114$. Exactement 21 femmes et 8 hommes. 
La première vision de Maria eut lieu le 9 août 1931, alors que les journaux commençaient à se lasser des apparitions. [...] La presse ne la mentionna qu'une seule fois bien qu'elle ait eu 139 visions entre le 9 août et le 13 décembre (la comptabilité était, semble-t-il, investie d'une valeur religieuse ; les voyants tenaient le compte de leurs visions tout comme les "Alliées" ${ }^{29}$ le faisaient pour leurs rosaires et leurs mortifications). Maria n'atteignit une certaine renommée que lorsque les interdictions de l'Église eurent décimé les rangs des voyants et les croyants. Elle continua à se rendre à Ezkioga durant plusieurs années et eut des visions jusqu'à sa mort, en 1950, tant à Ezkioga que chez elle ou dans d'autres endroits. ${ }^{30}$

Dans son article de 1998, Christian suggère que l'écart constaté à Ezkioga entre les voyants "auxquels on a cru» et ceux que l'on a "ignorés" est sans doute un fait général. Dans le passé comme dans le présent, certaines personnes ont pu croire qu'elles avaient vu la Vierge et taire leur expérience parce qu'elles pensaient que personne ne les croirait ; d'autres, qui ont vu leur récit récusé par leurs proches, ont renoncé à le diffuser audelà de ce premier cercle. L'historien ne peut donc affirmer, en toute rigueur, que les hommes étaient plus enclins que les femmes, au Moyen Âge, à voir la Vierge et que le XIX ciècle a inversé cette tendance. Mais on peut faire l'hypothèse que les critères sur lesquels se sont appuyés les populations et l'Église - celle-ci étant généralement plus sévère que les fidèles - pour décider de la crédibilité des voyants, se sont modifiés dans l'intervalle.

\section{Les jeunes filles et la Vierge}

La modification n'a guère affecté, comme on l'a vu, le cas des femmes mariées. Il en va tout autrement pour les fillettes et les jeunes filles : le renversement du sex ratio entre le Moyen Âge et la période contemporaine est dû à leur entrée massive sur la scène des apparitions. Cela est vrai au XIX ${ }^{e}$ siècle et le reste dans l'Espagne des années 1930. Lors des apparitions d'Ezkioga, les journaux parlèrent surtout des filles et elles furent aussi les

29 Les membres de la Alianza en Jesus por Maria, une pieuse association féminine.

30 Christian $1997: 106$. 
plus nombreuses à multiplier les expériences visionnaires : le groupe des "habitual seers" comprend huit hommes, vingt-deux garçons, vingt et une femmes et cinquante filles ${ }^{31}$. L'écart constaté, pour ce qui concerne les femmes mariées, entre le nombre des visionnaires et celles dont on parla dans la presse n'existe donc pas dans le cas des fillettes et des jeunes filles. On peut au demeurant supposer, comme le fait Christian ${ }^{32}$, que l'intérêt que leur portaient les journalistes les encouragea à «voir».

Leur propension à avoir des visions peut également être mise en rapport avec certains aspects de la "féminisation du catholicisme». Celle-ci, comme l'a montré C. Langlois, s'est traduite entre autres par la multiplication des congrégations féminines. Leurs membres se consacrent majoritairement à l'enseignement : en 1876, en France, six filles scolarisées sur dix sont instruites par des religieuses; «à cette même date, les frères enseignants touchent à peine trois garçons sur $\mathrm{dix}^{33}{ }^{33}$. Dans les écoles congréganistes (auxquelles il faudrait ajouter les ouvroirs destinés à donner aux plus pauvres un minimum d'instruction et un "métier convenable à leur sexe»), les filles reçoivent, bien entendu, une éducation religieuse qui accorde une large place au culte marial. Après la première communion, qui accompagne pour la majorité des garçons et des filles la fin de la scolarité, celles-ci continuent d'être prises en charge par l'Église. On les encourage alors à se joindre aux «Enfants (ou Filles) de Marie», une congrégation créée après les apparitions de la Vierge à Catherine Labouré en 1830 : dans son ouvrage sur Lourdes, R. Harris souligne opportunément que cette association y comptait, au moment des apparitions, plus de 130 membres $^{34}$. Il n'est donc pas étonnant que les filles aient, plus que les femmes, les hommes et les garçons, souhaité voir la Vierge - et que beaucoup aient effectivement fini par la voir.

L'Église se préoccupait d'encadrer les filles, mais aussi de les mettre en valeur. Les cérémonies et l'iconographie de la première communion sont

31 Christian 1998 : 114. L'auteur ne précise pas la proportion de fillettes et de jeunes filles.

32 Christian $1997: 252$.

33 Langlois 1991 : 293.

34 Harris 2001: 71. 
centrées sur les communiantes, toutes de blanc vêtues ${ }^{35}$. On voit aussi ces «filles blanches», communiantes de l'année ou Filles de Marie, dans toutes les cérémonies religieuses. Au début du $\mathrm{XX}^{\mathrm{e}}$ siècle, dans les missions paroissiales du Pays basque, celles-ci «reçoivent la communion après les enfants, ce qui les fait apparaître comme le groupe qui est, après ceux-ci, le plus proche de Dieun ${ }^{36}$. Ce contexte rituel contribue à expliquer que l'on ait accordé une créance particulière aux visions de ces figures angéliques.

On ne saurait oublier, cependant, que ces rituels exaltent un trait que l'Église a valorisé bien avant l'époque de la «féminisation du catholicisme» : la virginité féminine. Dans son livre sur la sainteté féminine, J.-P. Albert a souligné que les «vierges représentent environ $75 \%$ des saintes et des bienheureuses») et montré que la virginité a, pour les candidates à la sainteté, un poids symbolique qu'elle n'a absolument pas pour leurs homologues masculins $s^{37}$. Pour l'Église, certes, le fait de bénéficier d'une apparition n'est pas un critère de sainteté et elle n'a jamais canonisé une simple visionnaire avant le $\mathrm{XX}^{\mathrm{c}}$ siècle $^{38}$. Les clercs (et les fidèles avec eux), estiment cependant qu'une "vraie» apparition ne saurait être accordée qu’à des personnes dotées de certaines vertus. La virginité, pour les femmes, en fait partie. On ne saurait comprendre, autrement, que les filles soient bien plus nombreuses que les femmes dans les apparitions et ce, depuis la fin du Moyen Âge. Et c'est certainement parce que la valeur attachée à la virginité a beaucoup faibli qu'on voit, à partir des années 1960, les femmes mariées entrer sur la scène des apparitions ${ }^{39}$.

La place des jeunes filles dans les apparitions du XIX ${ }^{e}$ siècle est enfin liée, comme on l'a souvent dit, à l'aura de la figure de Bernadette. Les raisons de sa popularité sont complexes. Elles tiennent, pour une part, à sa personnalité, qui a fasciné tous ceux qui l'ont approchée. La publicité donnée au personnage (via la photographie notamment) s'explique, par

35 Delumeau : 1987.

36 Christian $1997: 252$.

37 Albert $1997: 28$.

38 Bernadette, qui était devenue entre temps, il est vrai, sœur Marie-Bernard, le fut en 1933.

39 Christian 1998. 
ailleurs, par la volonté des "promoteurs» de Lourdes de faire du sanctuaire le lieu de pèlerinage emblématique des catholiques français ${ }^{40}$. Il me semble, enfin, que la valorisation de Bernadette tient à deux caractéristiques de sa "façon de voir" la Vierge. Pour la première fois dans l'histoire des apparitions, les visions de Bernadette se produisirent en public et, chaque fois qu'elles eurent lieu, la voyante entra en extase ${ }^{41}$. Ceci est sans doute lié à cela : il fallait bien que Bernadette montre aux spectateurs qu'elle voyait la Vierge. La fillette se comporta donc comme les mystiques que l'on venait contempler durant leurs visions ${ }^{42}$. On conçoit, par conséquent, que les Lourdais l'aient très vite considérée comme une sainte. On conçoit aussi que les fillettes et les jeunes filles qui ont vécu dans les décennies suivantes aient pu souhaiter reproduire son expérience.

La place que prennent les jeunes filles dans les apparitions mariales du XIX ${ }^{c}$ siècle et des premières décennies du suivant peut sans doute être perçue comme un indice de la continuité des représentations de la femme dans le catholicisme : les seules femmes que l'Église consent à valoriser religieusement, à cette époque comme dans les siècles antérieurs, sont les vierges. Il me semble cependant que l'Église - et la société avec elle - a donné à la virginité féminine, au XIX siècle, un poids sans précédent : en témoigne la place donnée au blanc dans les costumes rituels, ceux des communiantes, des Enfants de Marie et des mariées. L'iconographie de l'Immaculée Conception montre aussi une Vierge (une très jeune fille qui n'est plus une Vierge-Mère) vêtue de blanc. Or, c'est précisément cette Vierge-là que voit Bernadette à Lourdes. Ce lien privilégié entre la Vierge et les jeunes filles s'est, sans conteste, distendu aujourd'hui.

40 Harris 2001.

41 Christian $1997: 22$.

42 Voir sur ce point Albert 1997 : ch. 8. 


\section{Bibliographie}

ALBERT Jean-Pierre, 1997, Le sang et le Ciel. Les saintes mystiques dans le monde chrétien, Paris, Aubier.

ALBERT LLORCA Marlène, 1995, "Les fils de la Vierge. Broderie et dentelle dans l'éducation des jeunes filles", L'Homme, 133, janv.-mars : 99-122.

BOUFLET Joachim et BOUTRY Philippe, 1997, Un signe dans le ciel. Les apparitions de la Vierge, Paris, Grasset.

CHIRON Yves, 1995, Enquête sur les apparitions de la Vierge, Paris, Eds. J'ai lu.

CHRISTIAN William A. Jr., 1981, Apparitions in Late Medieval and Renaissance Spain, Princeton, Princeton University Press (1990, Apariciones en Castilla y Cataluña, siglos XIV-XVI, trad. Eloy Fuente, Madrid, Nerea).

--, 1984, "Religious Apparitions and the Cold War in Southern Europe», in Eric R. Wolf ed., Berlin, Mouton : 239-266.

—, 1992, Moving Crucifixes in Modern Spain, Princeton-New Jersey, Princeton University Press.

—, 1996, Visionaries. The Spanish Republic and the Reign of Christ, Berkeley-Los Angeles, University of California Press (Las visiones de Ezkioga. La Segunda Republica y el Reino de Cristo, Barcelona, Ariel).

—, 1998, "Six Hundred Years of Visionaries in Spain : Those believed and Those ignored", in Michael Hanagan, Leslie Page Moch and Wayne Brake eds, Challenging Authority : The Historical Study of Contentious Politics, Minneapolis, University of Minnesota Press : 107-119.

DELUMEAU Jean (dir.), 1987, La première communion. Quatre siècles d'histoire, Paris, Desclée de Brouwer.

HARRIS Ruth, 2001, Lourdes. La grande histoire des apparitions, des pélerinages et des guérisons, trad. de Marie-Lise Hiaux-Heitzmann, Paris, J.-C. Lattès (Lourdes. Body and Spirit in the Secular Age, 1999, Allen Lane The Penguin Press).

LAMEIRE Gilles, 1978, Apparitions en Alsace. Notre-Dame de Neubois, chez l'auteur.

LANGLOIS Claude, 1991, "Féminisation du catholicisme», in Jacques Le Goff et René Rémond (dir.), Histoire de la France religieuse, t. 3, Du roi très chrétien à la lä̈cité républicaine (XVIIT-XIX' s.), Paris, Eds. du Seuil, pp. 292-310.

MAITRE Jacques, 1997, Mystique et féminité. Essai de psychanalyse sociohistorique, Paris, Eds. du Cerf.

VAUCHEZ André, 1999, Saintes, prophètes et visionnaires. Le pouvoir surnaturel au Moyen Âge, Paris, Albin Michel. 